\title{
Cyclin-Dependent Kinase 4 and Cyclin D1 Are Required for Excitotoxin-Induced Neuronal Cell Death In Vivo
}

\author{
Hidetoshi Ino and Tanemichi Chiba \\ Department of Neurobiology (C1), Graduate School of Medicine, Chiba University, 1-8-1 Inohana, Chuo-ku, Chiba 260- \\ 8670, Japan
}

Systemic administration of the glutamic acid analog kainic acid (KA) causes neuronal cell death in brain-vulnerable regions, such as the piriform cortex, hippocampus, and amygdala in rats. We investigated the relationship between the KA-induced neuronal apoptosis and expression of cyclin-dependent kinase 4 (CDK4) and cyclin D1, key regulators of cell cycle progression. Expression of CDK4 and cyclin D1 was upregulated in neurons of the rat piriform cortex and amygdala 1-3 d after KA administration in vivo. CDK4 and cyclin D1 proteins were induced in the cytoplasm and nuclei of neurons, with a concomitant increase of CDK4- and cyclin D1-positive microglia in the affected areas. Continuous infusion of $100 \mu \mathrm{m}$ CDK4 or cyclin D1 antisense oligonucleotides into the lateral ventricle using mini-

Several factors are involved in the induction of neuronal cell death (apoptosis) including activator protein-1 (AP-1) transcription factors (c-Fos and c-Jun), c-Jun-N-terminal kinase-3, caspases, the Bcl-2 family, and Akt kinase (for review, see Bergeron and Yuan, 1996; Pettmann and Henderson, 1998). In addition, several lines of evidence suggest a relationship between the cell cycle and apoptosis both in non-neuronal and neuronal cells. It is generally believed that DNA-damaged proliferating cells stop the cell cycle progression and conduct DNA repair; otherwise cell death occurs. Ectopic expression of cell cycle regulatory proteins, such as CDC2 (Shi et al., 1994), leads cells to apoptosis. In the nervous system, although most of the cells escape from the cell cycle, upregulation of cyclin D1 has been reported in dying neurons (Freeman et al., 1994; Kranenburg et al., 1996; Timsit et al., 1999), and its overexpression also induces neuronal cell death (Kranenburg et al., 1996). Cyclin D1, a member of the $G_{1}$ cyclins, plays an important role in the $G_{1}$ phase progression of the cell cycle in proliferating cells via activation of cyclin-dependent kinase 2 (CDK2), CDK4, or CDK6. In addition, chemical inhibitors of CDKs and expression of dominantnegative forms of CDK4 and CDK6 suppress the death of nerve growth factor-deprived PC12 cells and cultured sympathetic neurons (Park et al., 1996, 1997). However, little is known about the function of these cell cycle regulators in neuronal cell death in vivo.

In rats, systemic administration of the potent excitotoxin kainic acid (KA) causes delayed neuronal cell death in vulnerable regions of the brain, such as the piriform cortex, amygdala, anterior

Received Dec. 27, 2000; revised May 30, 2001; accepted June 1, 2001.

Correspondence should be addressed to Dr. Hidetoshi Ino, Department of Neurobiology (C1), Graduate School of Medicine, Chiba University, 1-8-1 Inohana, Chuo-ku, Chiba 260-8670, Japan. E-mail: ino@med.m.chiba-u.ac.jp.

Copyright (ㄷ) 2001 Society for Neuroscience $\quad 0270-6474 / 01 / 216086-09 \$ 15.00 / 0$ osmotic pumps suppressed the excitotoxin-induced neuronal cell death in the piriform cortex and basolateral amygdaloid nucleus, whereas sense oligonucleotides exhibited no such effect. Although KA administration causes prolonged c-Fos expression in the vulnerable regions that preceded the induction of neuronal apoptosis, the CDK4 or cyclin D1 antisense oligonucleotides exhibited no suppressive effect on C-Fos levels. Our results suggest that CDK4 and cyclin D1 are essential for KA-induced neuronal apoptosis in vivo.

Key words: CDK4; cyclin D1; cyclin-dependent kinases; cyclins; neuronal cell death; kainate; phosphorothioate antisense oligonucleotides olfactory nucleus, hippocampus, septum, and thalamus, whereas the cerebellum and brainstem are spared (Schwob et al., 1980). We used this system as a model to investigate the physiological significance of CDKs and cyclins in the induction of neuronal cell death in vivo.

\section{MATERIALS AND METHODS}

Animal experiments. Male Sprague Dawley rats (7-8 weeks old; 230-270 $\mathrm{gm})$ received an intraperitoneal injection of KA $(10 \mathrm{mg} / \mathrm{kg}$; Sigma, St. Louis, MO) dissolved in saline with $\mathrm{pH}$ adjusted with an equal mole of $\mathrm{NaOH}$. Control rats received intraperitoneal injections of saline alone. Alzet mini-osmotic pumps (model 2001) and brain infusion kits (Alza Pharmaceuticals, Palo Alto, CA) containing $100 \mu \mathrm{M}$ phosphorothioate oligonucleotides (custom synthesized by Amersham Pharmacia Biotech, Arlington Heights, IL) in saline were implanted into rats according to the manufacturer's protocol under sodium pentobarbital anesthesia $16 \mathrm{hr}$ before KA administration. Tips of the brain infusion kits were positioned in the right lateral ventricle $(-0.5 \mathrm{~mm}$ from bregma and $2.0 \mathrm{~mm}$ lateral). Oligonucleotide solution was continuously infused into the lateral ventricle at the rate of $1 \mu \mathrm{l} / \mathrm{hr}$. Nucleotide sequences of rat CDK4 and cyclin D1 antisense oligonucleotides were 5'-GTGGTAGCCATTCTCG-3' and 5'-GGAGCTGGTGTTCCAT-3', respectively.

Northern blot analysis. Experiments were performed as described previously (Ino et al., 1994). Mouse CDK4 and cyclin D1 cDNAs containing the full length of the coding regions were prepared using reverse transcriptase-PCR, cloned into pBluescript II vector (Stratagene, La Jolla, CA), and confirmed by sequencing. Nucleotide sequences of mouse CDK4 and cyclin D1 cDNAs within the regions used were 95 and 96\% identical to those of rat, respectively.

In situ hybridization, immunohistochemistry, and lectin histochemistry. Tissue was prepared as described previously (Ino et al., 1994; Ino and Chiba, 1996). Briefly, rats were perfused with $4 \%$ paraformaldehyde in $0.1 \mathrm{~m}$ phosphate buffer, $\mathrm{pH}$ 7.5. Brains were sliced at 5-7 mm thickness and were further fixed in the same solution at $4^{\circ} \mathrm{C}$ for $2 \mathrm{~d}$. The brains were then transferred to distilled water and incubated at $4{ }^{\circ} \mathrm{C}$ overnight. The brains were boiled in distilled water for $5 \mathrm{~min}$ and then immersed in $30 \%$ sucrose in PBS at $4^{\circ} \mathrm{C}$ overnight. The brains were frozen in dry ice, and cryostat sections $(10 \mu \mathrm{m})$ were prepared. Frozen sections, used for in situ hybridization, immunohistochemistry, and lectin histochemistry, 
were prepared by the identical procedure. In situ hybridization and immunohistochemistry were performed as described previously (Ino and Chiba, 1996). Primary antibodies for immunohistochemistry were the following: rabbit polyclonals anti-CDK4 (C-22; Santa Cruz Biotechnology, Santa Cruz, CA; 1:1000), anti-cyclin D1 (H-295; Santa Cruz Biotechnology; 1:1000), anti-c-Fos (Ab-5; Oncogene Research Products, Cambridge, MA; 1:2000), and anti-Egr-1 (C-19; Santa Cruz Biotechnology; 1:1000); goat polyclonal anti-FosB (102; Santa Cruz Biotechnology; 1:1000); and mouse monoclonals anti-neuronal nuclei (NeuN; Chemicon, Temecula, CA; 1:1000) and anti-glial fibrillary acidic protein (GFAP; G5A; Chemicon; 1:100). For single staining, sections were incubated in primary antibodies, followed by biotin-conjugated anti-rabbit $\operatorname{IgG}(\mathrm{H}+\mathrm{L})$ secondary antibody (Vector Laboratories, Burlingame, CA) and the Vectastain ABC kit (Vector Laboratories). Reaction was developed with 3,3'-diaminobenzidine (DAB) and hydrogen peroxide. For double staining, after incubation with primary antibodies, sections were incubated with biotin-conjugated anti-rabbit $\operatorname{IgG}(\mathrm{H}+\mathrm{L})$ antibody (Vector Labora-

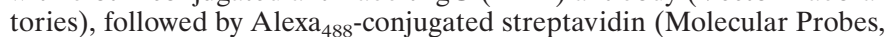
Eugene, OR) and Texas Red-conjugated anti-mouse IgG $(\mathrm{H}+\mathrm{L}$; Vector Laboratories). Sections were mounted with PermaFluor aqueous mounting medium (Shandon, Pittsburgh, PA) and observed by fluorescence microscopy. Griffonia simplicifolia lectin $\mathrm{I} \mathrm{B}_{4}$ subunit (GS I-B ${ }_{4}$; Vector Laboratories) was used to detect microglia. Digoxigenin (DIG)conjugated GS I- $\mathrm{B}_{4}$ was prepared using the DIG protein-labeling kit (Roche Diagnostics) according to the manufacturer's protocol. Sections were incubated with DIG-conjugated GS I-B $4(15 \mu \mathrm{g} / \mathrm{ml})$, reacted with rhodamine-conjugated sheep polyclonal anti-DIG Fab fragments (Roche Diagnostics), and observed by fluorescence microscopy.

Terminal deoxynucleotidyl transferase-mediated biotinylated-dUTP nickend labeling. Apoptosis was detected in the frozen sections prepared by the procedure described above. Sections were treated with $3 \%$ Triton $\mathrm{X}-100$ in PBS for $3 \mathrm{hr}$ at room temperature (RT). Sections were then immersed in terminal deoxynucleotidyl transferase-mediated biotinylated-dUTP nick-end labeling (TUNEL) buffer (30 mM Tris-HCl, $\mathrm{pH} 7.2,140 \mathrm{~mm}$ sodium cacodylate, $1 \mathrm{~mm}$ cobalt chloride, and $0.05 \%$ Tween 20) for $10 \mathrm{~min}$ at RT and reacted with $1.6 \mu \mathrm{M}$ biotin-16-dUTP (Roche Diagnostics) and $50 \mathrm{U} / \mathrm{ml}$ terminal deoxynucleotidyl transferase (TdT; Takara, Tokyo, Japan) in the TUNEL buffer for $1.5 \mathrm{hr}$ at $37^{\circ} \mathrm{C}$. After washing with PBS, sections were reacted with the Vectastain ABC kit and stained with DAB and hydrogen peroxide. For double TUNEL immunostaining, 1.6 $\mu \mathrm{M}$ DIG-11-dUTP (Roche Diagnostics) was used as a substrate for TdT. After washing with PBS and blocking with 5\% skim milk in PBS, immunohistochemistry was performed. The incorporated DIG was detected with rhodamine-conjugated anti-DIG Fab fragments.

Detection of oligonucleotide delivery. By the use of mini-osmotic pumps and brain infusion kits, $100 \mu \mathrm{M} 5^{\prime}$-amino-3'-biotin-labeled cyclin D1antisense phosphorothioate oligonucleotide (custom synthesized by Amersham Pharmacia Biotech) in saline was infused into the rat lateral ventricle. Rats were decapitated $2.5 \mathrm{~d}$ after the start of infusion, brains were immediately frozen in dry ice, and unfixed cryostat sections $(10 \mu \mathrm{m})$ were prepared. After drying, sections were fixed with $4 \%$ paraformaldehyde and $2 \%$ glutaraldehyde in $0.1 \mathrm{M}$ sodium phosphate, $\mathrm{pH} 7.5$, at RT for $30 \mathrm{~min}$. After washing with PBS, endogenous peroxidase was inactivated with $0.1 \%$ sodium azide and $0.3 \%$ hydrogen peroxide at RT for 10 min. After washing with PBS, reactivity to streptavidin was amplified using the Renaissance TSA-indirect kit (NEN Life Science Products, Boston, MA) according to the manufacturer's protocol. The incorporated biotin-labeled oligonucleotide was detected with Alexa ${ }_{488^{-}}$ conjugated streptavidin.

Count of TUNEL-positive cells. Stereotaxic coordinates and areas of the piriform cortex and basolateral amygdaloid nucleus were determined according to the rat brain atlas of Paxinos and Watson (1998). The molecular layer (layer I) was omitted from counted areas of the piriform cortex. TUNEL-positive cells per square millimeter on both sides of the piriform cortex $(-1.4,-2.3,-3.3$, and $-4.3 \mathrm{~mm}$ from bregma) and basolateral amygdaloid nuclei $(-2.3$ and $-3.3 \mathrm{~mm}$ from bregma) were counted.

\section{RESULTS}

\section{Upregulation of CDK4 and cyclin D1 expression after $\mathrm{KA}$ administration in the piriform cortex and amygdala}

Rats that received a single dose of KA exhibited typical behavioral changes. Arrest of movement was followed by hyperactiveness, trembling known as "wet dog shakes," head nodding, rear- ing, and convulsions. Seizures usually occurred $\sim 1 \mathrm{hr}$ after KA administration, and rats became highly aggressive $2-3 \mathrm{~d}$ after KA administration. Apoptosis, detected by TUNEL, was observed early in the piriform cortex and amygdala $1 \mathrm{~d}$ after KA injection and became most evident $2 \mathrm{~d}$ after KA administration, with a concomitant decrease of neurons detected with the neuronal marker NeuN (Mullen et al., 1992) (Fig. 1A,F). We performed in situ hybridization to examine expressional changes of cell cycle regulatory proteins by the use of antisense riboprobes and found that expression of CDK4 and cyclin D1 mRNA was significantly upregulated in these areas after KA administration (Fig. 1B,C). CDK4 expression was most evident $1 \mathrm{~d}$ after KA administration. Cyclin D1 expression was also most evident $1 \mathrm{~d}$ after KA administration. CDK4 and cyclin D1 expression was especially distinct in layer II neurons showing relatively large nuclei and cell bodies and compact layer formation in the piriform cortex (Fig. 1D,E). These neurons exhibited severe apoptosis after KA administration (see Figs. $1 A, 4 A$ ). Although in other regions it was not clear what class of cells expressed CDK4 and cyclin D1, some neurons seemed to be positive. No apparent signal was observed with sense probes (data not shown). Northern blot analysis further supported the observation of upregulation of CDK4 and cyclin D1 expression in the piriform and entorhinal cortices and amygdala (Fig. 2). In this case, the upregulation of CDK4 expression continued up to $3 \mathrm{~d}$ in these areas, whereas the cyclin D1 expression level was reduced $3 \mathrm{~d}$ after $\mathrm{KA}$ injection. It is likely that CDK4 mRNA in surviving neurons remains at elevated levels for several days after KA administration, although the number of neurons is greatly reduced. In addition, the contribution of microglia may not be negligible at later periods, as described below (Fig. $3 K, L$ ). Moderate levels of upregulation were also observed in the hippocampus, but not in the cerebellum or the cerebral cortex except for the piriform and entorhinal cortices.

\section{Immunohistochemistry of CDK4 and cyclin D1}

To identify the types of cells expressing CDK4 and cyclin D1, we performed an immunohistochemical study that showed changes in protein levels and subcellular localization. Because the anti-cyclin D1 antibody (H-295) showed partial cross-reactivity with cyclin D2 because of the close similarity of the amino acid sequences between cyclins D1 and D2, which was tested using glutathione $S$-transferase-fusion proteins of cyclins D1, D2, and D3 (data not shown), the possibility exists that cyclin D2 may have contributed to the immunoreactivity. We describe here $\mathrm{H}-295$ reactivity as simply cyclin D1 immunoreactivity, although we do not completely neglect the possible contribution of cyclin D2.

First, we show here that CDK4 and cyclin D1 are located in NeuN-positive neurons. Several, but not all, neurons showed weakto-moderate levels of CDK4 immunoreactivity especially in the amygdala, hippocampus, and hypothalamic nuclei in the normal rat brain. This signal was located in the cytoplasm of neurons, whereas most of the neuronal nuclei were devoid of staining (Fig. 3A). However, KA administration caused an apparent nuclear accumulation of CDK4 in neurons of the piriform cortex $1 \mathrm{~d}$ after injection (Fig. 3B). Coexistence of CDK4 and NeuN immunoreactivity was seen. Cytoplasmic staining was also more distinct in these neurons. This type of nuclear staining was also observed in the amygdala but was not clear in the other parts of the cerebral cortex. Although $2 \mathrm{~d}$ after KA administration the number of CDK4-positive neurons was reduced, some neurons still exhibited CDK4 immunoreactivity (Fig. 3C, white arrowheads). In the hippocampus, some pyramidal neurons showed CDK4 immunoreactivity in this period, although 


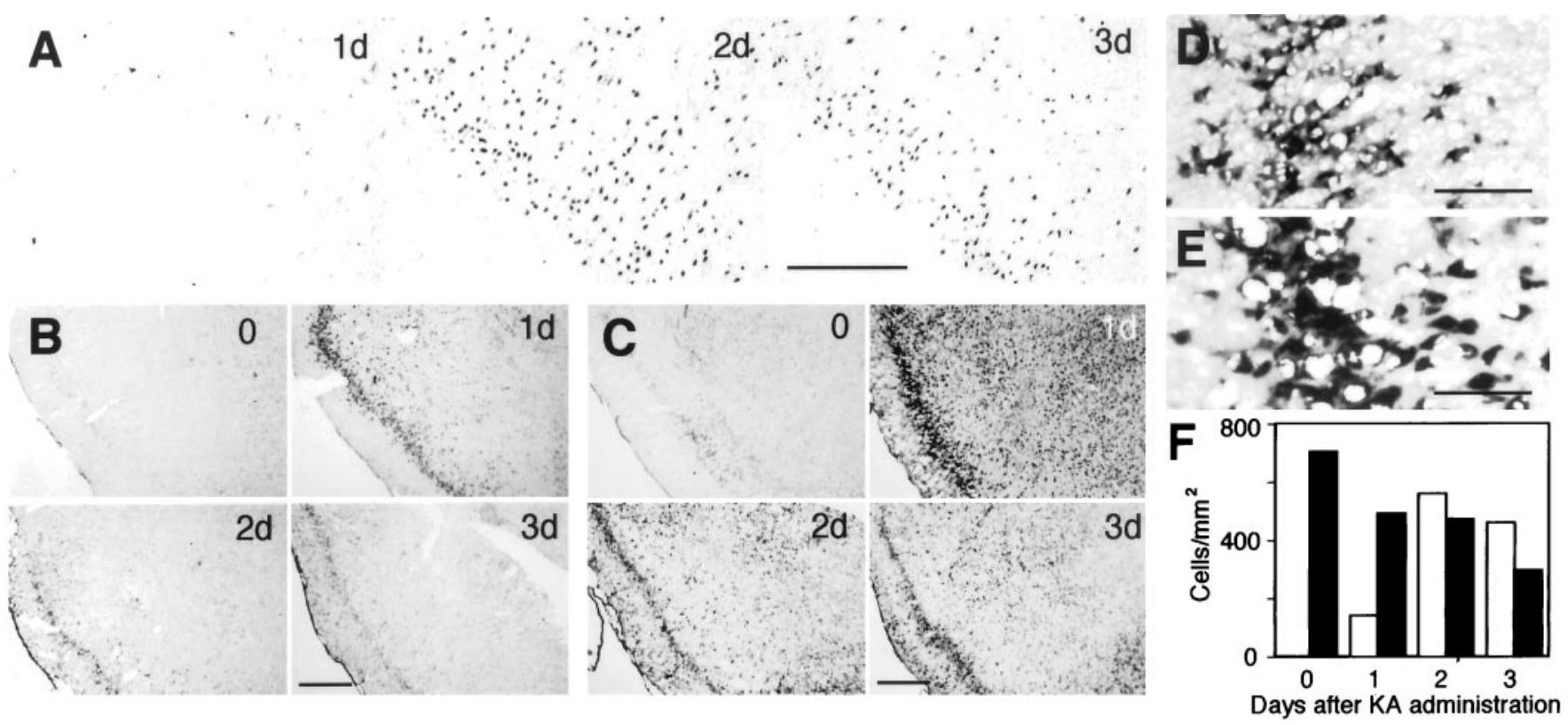

Figure 1. A, Neuronal cell death in the piriform cortex 1, 2, and $3 \mathrm{~d}$ after KA administration. Apoptotic cells were visualized by TUNEL. $B$, $C$, Upregulation of CDK4 $(B)$ and cyclin D1 $(C)$ expression in the piriform cortex and amygdala before (0) and 1, 2, and $3 \mathrm{~d}$ after KA administration detected by in situ hybridization. $D, E$, Higher magnification views of the CDK4 $(D)$ and cyclin D1 $(E)$ expression seen in $B$ and $C$ in layer II neurons of the piriform cortex $1 \mathrm{~d}$ after KA administration. Scale bars: $A, 200 \mu \mathrm{m} ; B, C, 500 \mu \mathrm{m} ; D, E, 100 \mu \mathrm{m}$. F, Temporal changes of TUNEL-positive (open columns) and NeuN-positive (closed columns) cells in the piriform cortex. The numbers of TUNEL-positive cells were counted in the sections shown in $A$ (approximately at the $1.4 \mathrm{~mm}$ caudal position from bregma). The numbers of NeuN-positive cells were counted in adjacent sections stained by immunohistochemistry.

Figure 2. Northern blot analysis of CDK4 $(A)$ and cyclin D1 $(B)$ expression in the rat brain before and after KA administration. Total RNA $(5 \mu \mathrm{g} /$ lane $)$ was analyzed. $P C+A m$ contains ventral areas under the line connecting the rhinal fissure and the optic tract and caudal areas from the optic chiasma. This tissue contains the piriform and entorhinal cortices and the amygdala. $C X$ contains areas of the cerebral cortex over the rhinal fissure (devoid of the piriform and entorhinal cortices). E12 is used as the positive controls. Upregulation of CDK4 and cyclin D1 mRNA expression was observed strongly in $P C+A m$ and slightly in $H i, 1-3$ d after KA administration. No apparent change was found in $C X$ and $\mathrm{Cb}$. Asterisks indicate glycerol-3phosphate dehydrogenase expression that served as a control. $C b$, Cerebellum; $C X$, cerebral cortex; E12, whole rat embryo at embryonic day $12 ; \mathrm{Hi}$, hippocampus; $P C+$ $A m$, piriform cortex- and amygdala-enriched area.
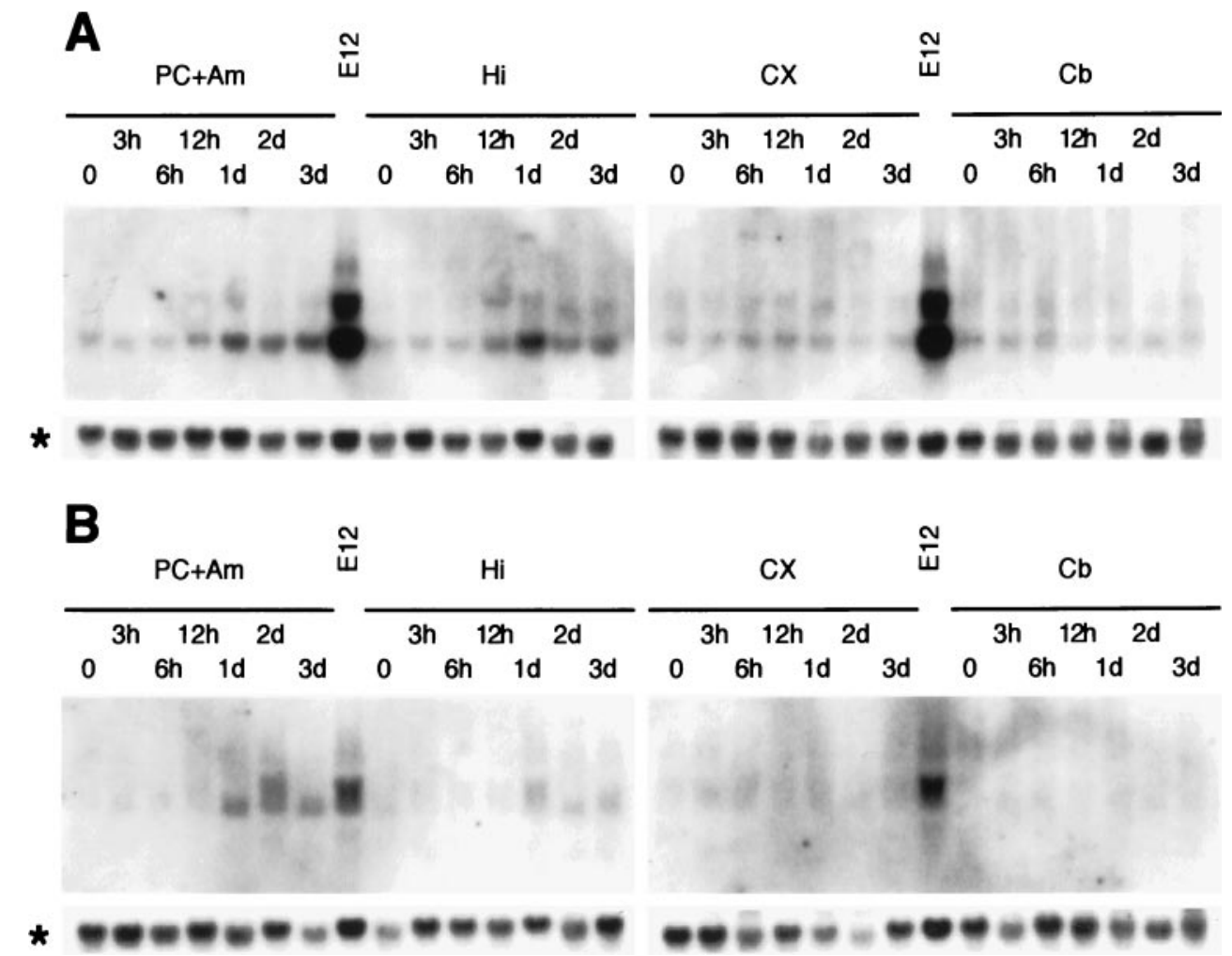

the level was lower than that in the piriform cortex (Fig. 3G). In contrast to CDK4, cyclin D1 immunoreactivity in the normal rat brain was low, although some neurons showed faint cytoplasmic staining (Fig. 3D). Cyclin D1 immunoreactivity in neurons of the piriform cortex was evident $1 \mathrm{~d}$ after KA administration (Fig. $3 E$ ).
Although intensity of this immunoreactivity tended to decrease $2 \mathrm{~d}$ after KA administration, many cyclin D1 and NeuN doublepositive neurons were still observed (Fig. $3 F$, white arrowheads). As was the case for CDK4, cyclin D1 was located both in the cytoplasm and nuclei of neurons. These changes were also observed in 

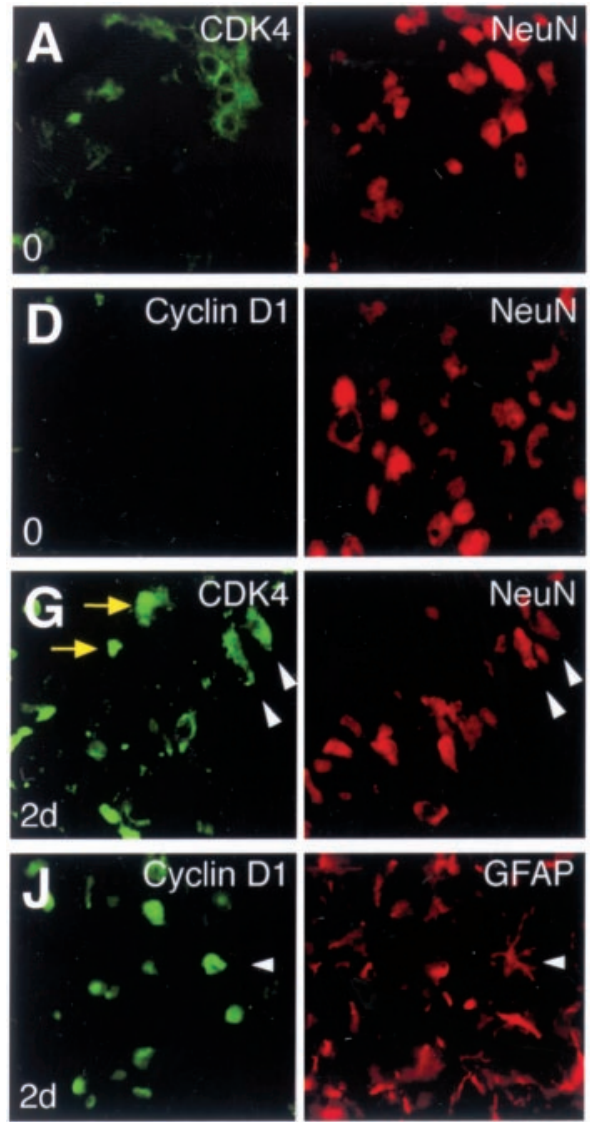
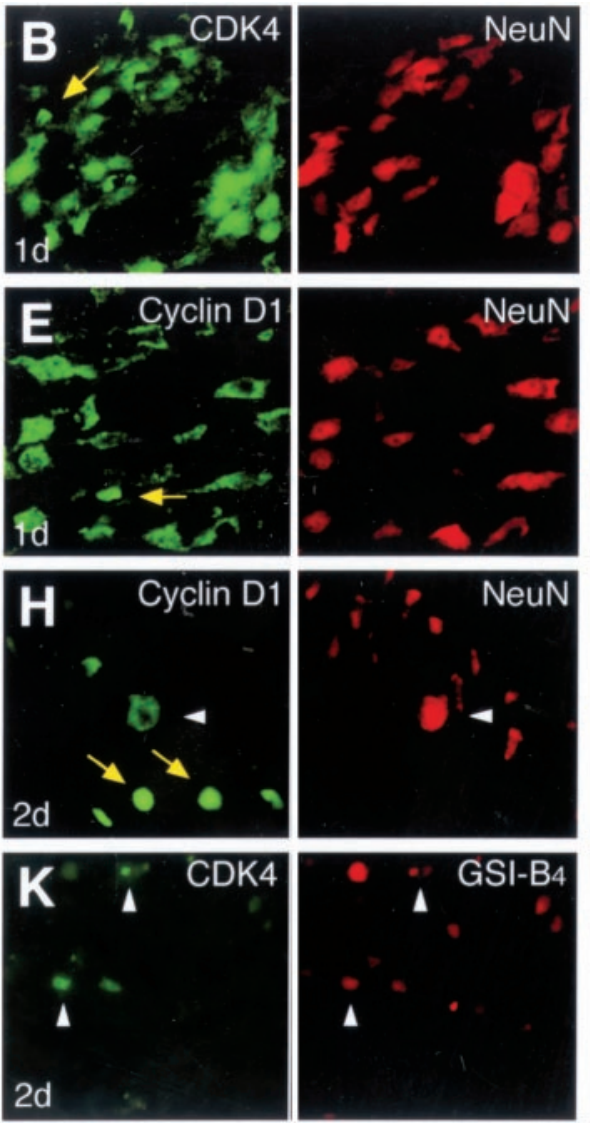
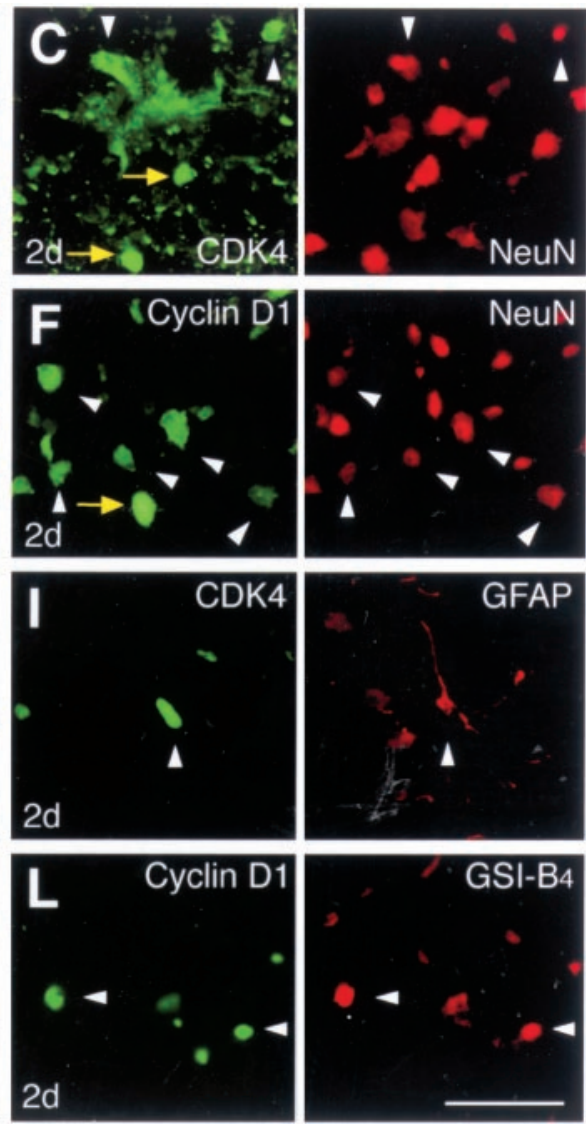

Figure 3. $A-F$, Induction of CDK4 $(A-C$; green $)$ and cyclin D1 $(D-F ;$ green $)$ in neurons of the piriform cortex before $(0 ; A, D)$ and $1 \mathrm{~d}(B, E)$ and $2 \mathrm{~d}$ $(C, F)$ after KA administration detected by immunohistochemistry. The intensity of CDK4 and cyclin D1 immunoreactivity increased $1 \mathrm{~d}$ after KA administration. The majority of CDK4- and cyclin D1-positive cells were also positive for the neuronal marker NeuN (red). CDK4 and cyclin D1 immunoreactivity was located in the cytoplasm, as well as in the nuclei. Although the number of CDK4-positive neurons tended to decrease $2 \mathrm{~d}$ after KA administration, some neurons still showed CDK4 staining $(C$; white arrowheads). CDK4- or cyclin D1-positive cells independent of NeuN are indicated by yellow arrows. $G, H$, Colabeling of CDK4 ( $G$; green) and cyclin D1 (H; green) with NeuN (red) in the CA1 hippocampal region $2 \mathrm{~d}$ after KA administration. CDK4- or cyclin D1-positive neurons are indicated by white arrowheads, and CDK4- or cyclin D1-positive non-neuronal cells are indicated by yellow arrows. I, J, Colabeling of CDK4 (I; green) and cyclin D1 (J; green) with GFAP (red) in the peripheral area of the piriform cortex $2 \mathrm{~d}$ after KA administration. Although CDK4 and cyclin D1 were observed in GFAP-positive astrocytes (white arrowheads), these cells were rarely found. $K, L$, Coexistence of CDK4 (K; green) and cyclin D1 (L; green) with GS I-B ${ }_{4}$ reactivity $($ red $)$, a marker of microglia in the basolateral amygdaloid nucleus, $2 \mathrm{~d}$ after KA administration. Strong nuclear CDK4 and cyclin D1 immunoreactivity was observed sporadically in microglia in the affected areas (white arrowheads). These microglia may be proliferating. Scale bar, $100 \mu \mathrm{m}$.

the amygdala but were not evident in the hippocampus and other parts of the cerebral cortex. Cytoplasmic cyclin D1 immunoreactivity was only occasionally observed in the hippocampal pyramidal neurons (Fig. 3H).

Although, as shown in Figure $3 A-H$, most of the CDK4 and cyclin D1 immunoreactivity was found associated with $\mathrm{NeuN}$ immunoreactivity, CDK4-positive NeuN-negative and cyclin D1-positive NeuN-negative cells also existed (yellow arrows), suggesting that CDK4 and cyclin D1 are also induced in glia. Then we examined this induction in glia, using the astrocytic marker GFAP and the G. simplicifolia lectin GS I-B $\mathrm{B}_{4}$ as a marker of microglia (Streit, 1990). GFAP immunoreactivity in the vulnerable regions was greatly reduced $2 \mathrm{~d}$ after KA administration, which may have been caused by arrested GFAP synthesis in surviving astrocytes rather than cell death of the astrocytes. Although astrocytes showing both CDK4 and GFAP or cyclin D1 and GFAP immunostaining were observed in the peripheral regions around the affected areas, these cells were rare (Fig. 3I,J). In contrast, CDK4- and cyclin D1-positive

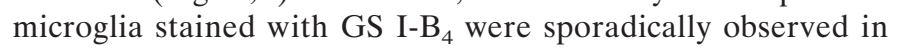

the affected areas (Fig. $3 K, L$ ). Although GS I-B -positive $^{-}$ microglia were sparse in the normal rat brain, they increased after KA administration. The number of these microglia was much smaller than that of neurons; however, CDK4 and cyclin D1 immunoreactivity was more intense and restricted to nuclei. In the experiments using 5-bromo-2'-deoxyuridine (BrdU) as a marker for cell proliferation, BrdU was incorporated into microglia but not into neurons 2-3 d after KA administration (data not shown), suggesting that CDK4- or cyclin D1-expressing microglia undergo cell cycle progression for cell proliferation. The peak of CDK4 and cyclin D1 expression was delayed by $1-2 \mathrm{~d}$ in microglia, compared with neurons. In addition to neurons, astrocytes, and microglia, CDK4- and cyclin D1-positive cells that showed no reactivity to the markers also existed.

\section{CDK4 and cyclin D1 immunoreactivity in TUNEL-positive cells}

Double TUNEL-immunolabeling experiments were performed to examine whether TUNEL staining was found associated with 

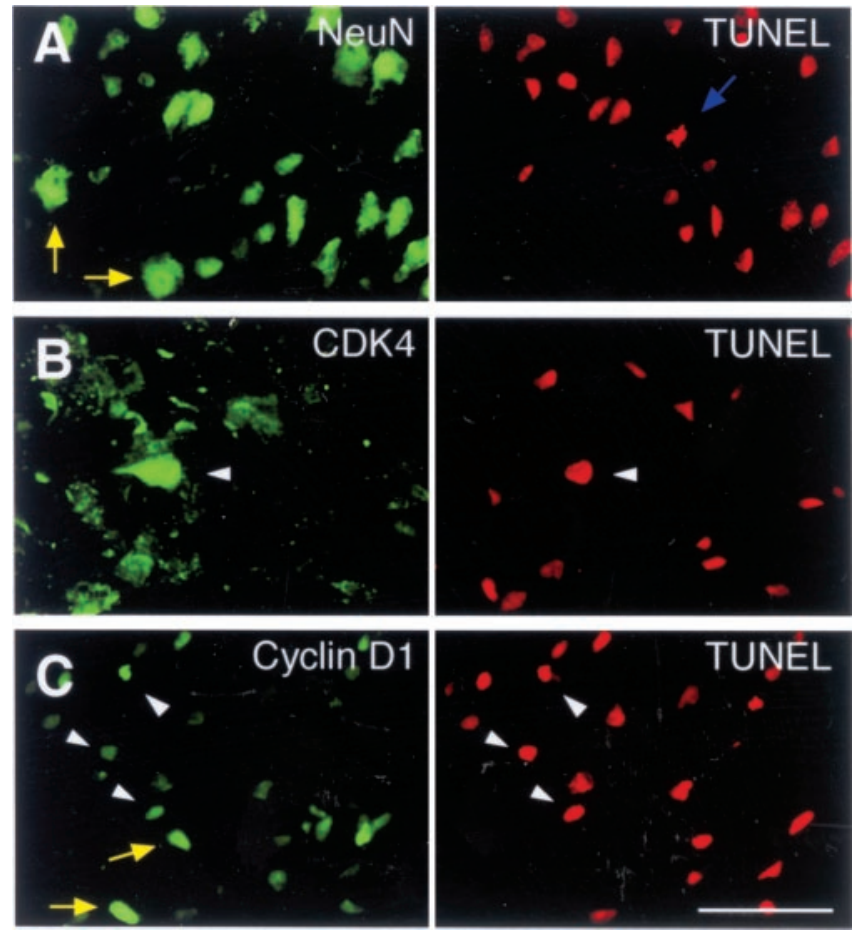

Figure 4. Coincidence of TUNEL reactivity (red) with NeuN (A; green), CDK4 $(B$; green $)$, and cyclin D1 $(C$; green $)$ immunoreactivity in the piriform cortex $2 \mathrm{~d}$ after KA administration. Although the majority of NeuN-positive neurons were TUNEL positive, TUNEL-negative (intact) neurons were also found ( $A$; yellow arrows). A pyknotic cell at the advanced stage of apoptosis lost NeuN immunoreactivity ( $A$; blue arrow). Most of the TUNEL-positive cells were devoid of CDK4 immunoreactivity, although a few cells showed TUNEL-CDK4 double staining ( $B$; white arrowheads). Although several TUNEL-cyclin D1 double-positive cells were observed ( $C$; white arrowheads), the cyclin D1 immunoreactivity was generally weak. Cyclin D1-positive TUNEL-negative cells also existed $(C$; yellow arrows). Scale bar, $100 \mu \mathrm{m}$.

CDK4 and cyclin D1 in dying cells. The majority of NeuNpositive neurons in the piriform cortex were TUNEL positive $2 \mathrm{~d}$ after KA administration (Fig. 4A). Although intact (TUNELnegative) neurons showed large nuclei and cell bodies (Fig. $4 A$, yellow arrows), TUNEL-positive neurons were shrunken, and pyknotic cells at the advanced stage of apoptosis lost NeuN reactivity (Fig. 4A, blue arrow). At this time, most TUNELpositive cells were CDK4 negative; only a few cells showed CDK4 immunoreactivity (Fig. 4B). Several TUNEL- and cyclin D1positive cells were also found in this period; however, cyclin D1 immunoreactivity of these cells was weak (Fig. 4C). No TUNEL reactivity was found in sporadic cells, likely to be microglia, showing intense CDK4 or cyclin D1 immunoreactivity.

\section{Suppression of neuronal cell death by infusion of CDK4 and cyclin D1 antisense oligonucleotides}

To determine whether there is a causal relationship between upregulation of CDK4 and cyclin D1 and induction of neuronal cell death in the rat brain, we performed in vivo infusion experiments using antisense oligonucleotides. An antisense or sense phosphorothioate oligonucleotide (100 $\mu \mathrm{M}$ in saline) against rat CDK4 or cyclin D1 was continuously infused into the rat lateral ventricle using osmotic pumps at the rate of $1 \mu \mathrm{l} / \mathrm{hr}$. Previous experiments have shown that infused phosphorothioate oligonucleotides are incorporated into cells and suppress translation of target proteins (Whitesell et al., 1993; Cui et al., 1999). First, to assess the delivery of oligonucleotides, amino- and biotin-labeled oligonucleotides were infused. Signal was detected in ependymal cells and presumably astrocytes around the ipsilateral (right) but not the contralateral (left) lateral ventricle (Fig. $5 A$ ), suggesting that oligonucleotides diffused only in the ipsilateral side. Incorporation of oligonucleotides was also observed into the third ventricle, but not into the ipsilateral and contralateral olfactory ventricles (data not shown). Rats displayed no apparent behavioral or histological alterations because of continuous infusion of either antisense or sense oligonucleotides. Rats received a KA injection $16 \mathrm{hr}$ after implantation of osmotic pumps, and apoptosis was determined by TUNEL $2 \mathrm{~d}$ after KA administration. Both antisense and sense oligonucleotide-inf used rats displayed a typical behavioral reaction after KA administration and became aggressive. Only rats that displayed these changes were used for further analyses.

In the CDK 4 antisense oligonucleotide-inf used brain $1 \mathrm{~d}$ after KA administration, CDK4 immunoreactivity in neurons of the piriform cortex on the ipsilateral side was weaker than that on the contralateral side (Fig. 5B). However, CDK4 immunoreactivity, especially in the cytoplasm, was not completely suppressed even on the ipsilateral side, which may have been caused by the ineffectiveness of antisense oligonucleotide on preexisting proteins or an insufficient concentration of oligonucleotide to suppress transcription completely. In the case of the cyclin D1 antisense oligonucleotide-infused brain, cyclin D1 immunoreactivity was similarly suppressed in neurons of the ipsilateral piriform cortex (Fig. $5 C$ ), although weak immunoreactivity on the ipsilateral side was observed, which may have been caused by an insufficient concentration of oligonucleotide or the cross-reactivity of the antibody to cyclin D2. In either case, the antisense oligonucleotides exhibited no suppressive effect on the upregulation of mRNA expression observed by in situ hybridization (data not shown).

In the CDK4 sense oligonucleotide-infused brain $2 \mathrm{~d}$ after KA administration, many apoptotic cells were observed on both sides of the piriform cortex and amygdala (Fig. 6). In contrast, in the CDK4 antisense oligonucleotide-infused brain, the number of apoptotic cells was greatly reduced in the ipsilateral piriform cortex and basolateral amygdaloid nucleus, whereas the contralateral side was unchanged from control levels (Figs. 5D, 6). Similar results were also obtained in the cyclin D1 antisense or sense oligonucleotide-infused brain (Figs. $5 E, 6$ ). In this case, the ipsilateral piriform cortex and basolateral amygdaloid nucleus of the cyclin D1 antisense oligonucleotide-infused brain were almost intact. However, in the olfactory bulb, anterior olfactory nucleus, thalamus, and hippocampus, apoptosis was not suppressed with either CDK4 or cyclin D1 antisense oligonucleotides. Protected neurons in the ipsilateral piriform cortex and amygdala of CDK4or cyclin D1-inf used brains survived 1 week after KA administration, at which time TUNEL reactivity was not observed (data not shown).

Prolonged expression of c-Fos caused by systemic KA administration is characteristic in vulnerable regions (Kasof et al., 1995). Expression of c-Fos reached a maximum level $12 \mathrm{hr}$ after KA administration, followed by a gradual decrease. The prolonged expression of c-Fos was observed both in the ipsilateral and contralateral piriform cortex and basolateral and medial amygdaloid nuclei infused with CDK4 and cyclin D1 antisense oligonucleotides, respectively (Fig. $5 F, G$ ). Prolonged upregulation of FosB and Egr-1 expression was also observed, and con- 

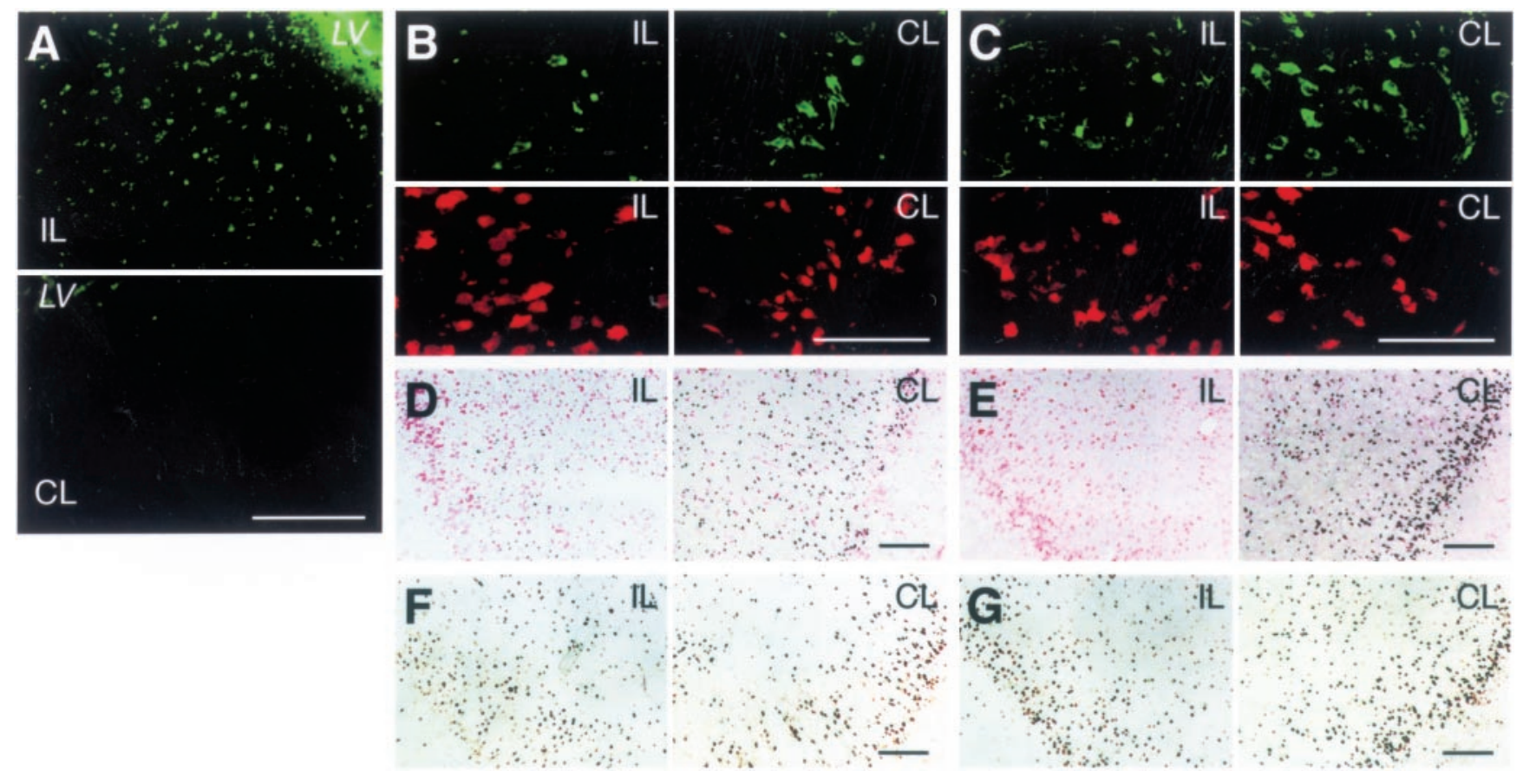

it

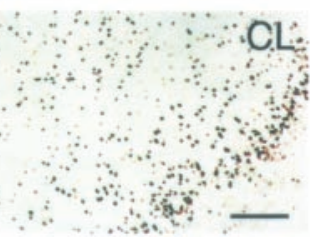

Figure 5. Suppression of neuronal cell death in the piriform cortex and amygdala by CDK4 or cyclin D1 antisense oligonucleotides. $A, 5^{\prime}$-Amino- $3^{\prime}$ biotin-labeled oligonucleotide was detected in cells around the ipsilateral but not the contralateral side of the lateral ventricle in the rat brain $2.5 \mathrm{~d}$ after the start of infusion. Staining was mainly found in the ependymal cells and presumably astrocytes. $B, C$, CDK4 immunoreactivity was suppressed by CDK4 antisense oligonucleotide (B; green), and cyclin D1 immunoreactivity was suppressed by cyclin D1 oligonucleotide $(C$; green) 1 d after KA administration. Suppression was observed in the ipsilateral but not the contralateral side of the piriform cortex. NeuN staining in the identical fields is also shown $(B, C ;$ red $) . D, E$, The number of TUNEL-positive cells was dramatically reduced in the ipsilateral but not contralateral piriform cortex and amygdala by inf usion of CDK4 $(D)$ or cyclin D1 $(E)$ antisense oligonucleotide $2 \mathrm{~d}$ after KA administration. Sections were counterstained with neutral red. $F, G$, Immunostaining of c-Fos in the piriform cortex and amygdala $12 \mathrm{hr}$ after KA administration is shown. No apparent change was observed by infusion of CDK4 $(F)$ or cyclin D1 $(G)$ antisense oligonucleotide. $C L$, Contralateral; $I L$, ipsilateral; $L V$, lateral ventricle. Scale bars: $A, D-G, 200 \mu$ m; $B, C, 100 \mu \mathrm{m}$.

tinuous infusion of antisense oligonucleotides did not influence their expression (data not shown).

\section{DISCUSSION}

\section{Involvement of CDK4 and cyclin D1 in neuronal cell death}

The present study showed that CDK4 and cyclin D1 are involved in the signaling pathway leading to apoptosis in neurons of the CNS after KA injection in vivo. Although growing evidence suggests a relationship between CDK4 and cyclin D1 and neuronal cell death, the question of whether they are essential for induction of apoptosis or are expressed to protect cells from apoptosis has yet to be clarified, although the former possibility has been strongly suggested. Upregulation of CDK4 and cyclin D1 in neurons is evident at the level of both mRNA and protein in the course of neuronal apoptosis. Although the CDK4 protein, the original function of which is unknown, is present in the cytoplasm of normal rat neurons, it is possible that newly synthesized CDK4 and cyclin D1 may be responsible for induction of apoptosis.

The possible relationship between cyclin D1 and neuronal apoptosis was first described in cultured neurons, and later it was shown that cyclin D1 expression was also upregulated in ischemia- and excitotoxin-induced neuronal cell death in vivo. There may exist a common mechanism in apoptosis induction regardless of the agents. Timsit et al. (1999) reported the cytoplasmic and nuclear localization of cyclin D1 in hippocampal neurons after ischemia, which closely resembles our observations in neurons of the piriform cortex and amygdala. However, there are some inconsistent points; we observed CDK4 and cyclin D1 immunoreactivity in presumably proliferating microglia, whereas they did not.

Our results indicate that not all of the KA-induced neuronal apoptosis is dependent on CDK4 and cyclin D1 expression. Neuronal cell death in the olfactory bulb, anterior olfactory nucleus, and thalamus was not suppressed by oligonucleotides, which may be caused by poor penetration of oligonucleotides into these areas at long distances from the injection point, as shown by the oligonucleotide delivery tests. In contrast, the hippocampus is located near the injection point. In our model, the CA1 region was one of the areas displaying severe cell death; however, neither CDK4 nor cyclin D1 antisense oligonucleotides suppressed neuronal cell death in this region, although upregulation of CDK4 expression was observed. One of the features of the KAinduced neuronal cell death observed in the CA1 is the delayed onset of cell death, which peaks from 3 to $4 \mathrm{~d}$ after KA administration when the number of apoptotic cells declines in the piriform cortex and amygdala. This implies that cell death in the CA1 is caused, not by a direct action of KA on neurons, but by an indirect effect via fiber connections. Cell death was not induced in the CA3 region except for the caudal part. It is also plausible that other factors substitute for CDK4. One of the best possible candidates is CDK6, playing similar roles to CDK4 in the cell cycle control; however, the role of CDK6 in neuronal cell death has remained unknown. Although the hippocampus is the area generally attracting the most attention in the study of neuronal 
Figure 6. Graphical representation of suppression of neuronal cell death with antisense oligonucleotides. TUNEL-positive cells in the ipsilateral $(I L)$ and contralateral $(C L)$ sides of the piriform cortex $(P C)$ and basolateral amygdaloid nucleus $(\mathrm{Am})$ infused with CDK4 (top) and cyclin D1 (bottom) antisense (open columns) and sense (closed columns) oligonucleotides $2 \mathrm{~d}$ after KA administration. The numbers of TUNEL-positive cells in the ipsilateral side of the piriform cortex were greatly reduced by infusion of CDK4 or cyclin D1 antisense oligonucleotides. TUNELpositive cells were counted in sections at the $1.4,2.3,3.3$, and $4.3 \mathrm{~mm}$ caudal positions from bregma. The molecular layer of the piriform cortex was excluded from the counting areas. Error bars indicate SD $(* * * p<0.01 ; * * p<$ $0.03 ; * p<0.05 ; n=3$; Welchi's $t$ test between antisense and sense oligonucleotide-infused tissues).
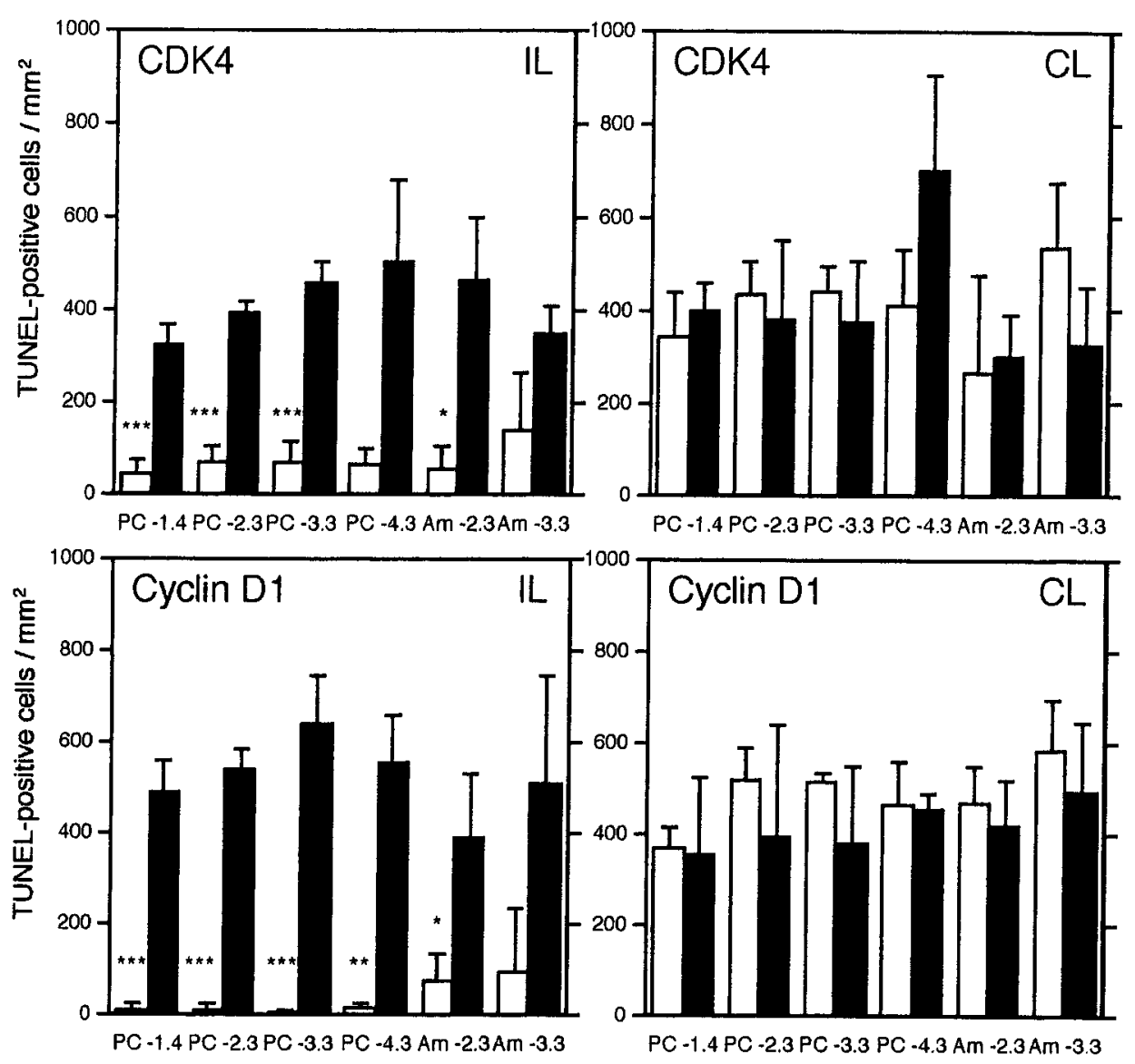

cell death, we did not focus on the hippocampus in our study because of the above-stated reasons.

The peak of CDK4 and cyclin D1 expression levels in neurons, 1-2 d after KA administration, preceded that of TUNEL reactivity, which reached the peak 2-3 d after KA administration. Although some TUNEL-positive neurons showed CDK4 or cyclin D1 immunoreactivity (Fig. 4), they were exceptional and most of the apoptotic neurons were CDK4 and cyclin D1 negative. These data suggest a time lag between CDK4 and cyclin D1 expression and the onset of DNA fragmentation, which is also supported by observations made by another group (Timsit et al., 1999).

\section{CDK4 and cyclin D1 protein levels in affected neurons}

Although multiple lines of evidence suggest that expression of cyclin D1 mRNA is upregulated in apoptotic neurons, there is no consensus concerning changes in protein levels. Increased levels of intensity of CDK4 and cyclin D immunoreactivity were observed in the Alzheimer's disease brain (McShea et al., 1997; Busser et al., 1998), in the rat brain after injury (Kaya et al., 1999), and in the rabbit spinal cord after ischemia (Sakurai et al., 2000). In contrast, cyclin D1 was observed in normal rat cortical neurons as a constitutive protein, and the levels of cyclin D1 were unchanged after induction of apoptosis in cultured embryonic neurons (Giovanni et al., 1999; Park et al., 2000). We believe that this discrepancy may originate from differences in the specificity of the antibodies used for detection. We tested several anti-cyclin D1 antibodies and found that some commercially available antibodies reacted with as yet unidentified proteins. For example, the R-124 mouse monoclonal antibody (Santa Cruz Biotechnology) reacted with great intensity with an unidentified protein $(29 \mathrm{kDa})$ in normal mouse and rat brain extracts. This protein is not cyclin $\mathrm{D} 1$, because the molecular weight of cyclin D1 is $36 \mathrm{kDa}$. Another mouse monoclonal antibody (DCS-6; Lab Vision, Fremont, CA) reacted with another unidentified protein $(25 \mathrm{kDa})$, and this antibody stained glial nuclei in the normal rat brain. We used the $\mathrm{H}-295$ antibody because it reacts only with proteins $(36 \mathrm{kDa})$ in mouse and rat embryo extracts and exhibits no reactivity in normal brain extracts, although it shows a partial cross-reactivity to cyclin D2. As shown here, protein levels of CDK4 and cyclin D1 are also upregulated in apoptotic neurons, and this observation is consistent with the increased levels of mRNA. However, we do not exclude the possible involvement of post-translational control of CDK4 and/or cyclin D1 by phosphorylation and/or dephosphorylation.

\section{Possible involvement of other cell cycle regulatory proteins in neuronal cell death and mechanisms of cell death}

Participation of other CDKs and cyclins in neuronal cell death must also be considered. We observed no apparent upregulation of $\mathrm{CDC} 2, \mathrm{CDK} 2$, and cyclin $\mathrm{A}, \mathrm{B} 1, \mathrm{~B} 2, \mathrm{D} 3$, and $\mathrm{E}$ expression in neurons after KA administration (H. Ino, unpublished observations). Slight upregulation of cyclin D2 and cyclin $G_{1}$ was observed in our preliminary experiments, but a detailed examination has yet to be performed (Ino, unpublished observations). Experiments concerning CDK6 have not been performed.

There is a hypothesis that postmitotic neurons die in an attempt to reenter the cell cycle in an uncoordinated and inappropriate manner. Retinal neurons show cell death in simian virus 40 
$\mathrm{T}$ antigen-transgenic mice (Hammang et al., 1993). Levels of proliferating cell nuclear antigen (PCNA, a protein necessary for DNA replication), cyclin $\mathrm{D}$, and $\mathrm{BrdU}$ incorporation are elevated in dying cerebellar granule cells of Purkinje cell-deficient mutant mice (Herrup and Busser, 1995). However, in our model we did not observe either BrdU incorporation or upregulation of PCNA in neurons except microglia, although a certain level of PCNA expression is observed in normal mature neurons (Ino and Chiba, 2000).

Further complicating this issue is the possible involvement of CDK inhibitors. In preliminary experiments, we have observed upregulation of $\mathrm{p} 21^{\text {Waf1/Cip } 1}$ in the piriform cortex and amygdala after KA injection. Freeman et al. (1994) have reported the upregulation of INK4 expression in neurons. Upregulation of p21 Waf1/Cip1 expression has also been observed in the ischemic rat hippocampus (van Lookeren Campagne and Gill, 1998). These CDK inhibitors may prevent neurons from undergoing apoptosis. Additionally, activation of CDKs (Zhou et al., 1998) and inactivation of p21 Waf1/Cip1 (Gervais et al., 1998; Levkau et al., 1998) by digestion with caspases have been reported in nonneuronal apoptotic cells.

The position that CDK4 and cyclin D1 occupy in the signaling pathway for induction of neuronal cell death and cross talk with other signaling pathways such as phosphatidylinositol 3-kinase and Akt kinase remains mostly unknown. c-Fos (Smeyne et al., 1993) and perhaps c-Jun (Estus et al., 1994; Schlingensiepen et al., 1994) and JNK3 (Yang et al., 1997) may be positioned upstream of CDK4 and cyclin D1, as is suggested by data showing that c-Fos expression precedes and is independent of CDK4 and cyclin D1 expression. The cyclin D1 promoter contains a consensus AP-1 site, and its induction is directly facilitated by c-Fos (Brown et al., 1998) and c-Jun (Wisdom et al., 1999) in the cell cycle progression in fibroblasts. Cyclin D1 may also be directly induced by AP-1 transcription factors in apoptotic neurons, although much evidence is needed to prove this possibility. As to downstream events, CDK4 and cyclin D phosphorylate Rb protein and p107 in the cell cycle progression, and involvement of $\mathrm{Rb}, \mathrm{p} 102$, and E2F-DP complexes has been reported in apoptotic cultured neurons (Giovanni et al., 1999; Park et al., 2000). Although this is an intriguing possibility, we have not yet detected phosphorylated $\mathrm{Rb}$ protein in our model. Evidence presented by us and others suggests that cyclin D1 participates in apoptosis by activating CDK4, although the possibility that cyclin D1 functions independently of CDKs cannot be ignored because the estrogen receptor is directly activated solely by cyclin D1 (Neuman et al., 1997; Zwijsen et al., 1997).

In a preclinical study of the treatment of stroke, targeting the dependency of neuronal apoptosis on cell cycle regulators was used to prevent reperfusion injury after ischemia (Osuga et al., 2000). The relationship between these factors and neuronal cell death is of importance both from a basic, as well as a clinical, viewpoint.

\section{REFERENCES}

Bergeron L, Yuan J (1996) Sealing one's fate: control of cell death in neurons. Curr Opin Neurobiol 8:55-63.

Brown JR, Nigh E, Lee RJ, Ye H, Thompson MA, Saudou F, Pestell RG, Greenberg ME (1998) Fos family members induce cell cycle entry by activating cyclin D1. Mol Cell Biol 18:5609-5619.

Busser J, Geldmacher DS, Herrup K (1998) Ectopic cell cycle proteins predict the sites of neuronal cell death in Alzheimer's disease brain. J Neurosci 18:2801-2807.

Cui J-K, Hsu CY, Liu PK (1999) Suppression of postischemic hippocampal nerve growth factor expression by a c-fos antisense oligonucleotide. J Neurosci 19:1335-1344.
Estus S, Zaks WJ, Freeman RS, Gruda M, Bravo R, Johnson Jr EM (1994) Altered gene expression in neurons during programmed cell death: identification of c-jun as necessary for neuronal apoptosis. J Cell Biol 127:1717-1727.

Freeman RS, Estus S, Johnson Jr EM (1994) Analysis of cell cyclerelated gene expression in postmitotic neurons: selective induction of cyclin D1 during programmed cell death. Neuron 12:343-355.

Gervais JLM, Seth P, Zhang H (1998) Cleavage of CDK inhibitor p21 $1^{\text {Cip1/Wafl }}$ by caspases is an early event during DNA damage-induced apoptosis. J Biol Chem 273:19207-19212.

Giovanni A, Wirtz-Brugger F, Keramaris E, Slack R, Park DS (1999) Involvement of cell cycle elements, cyclin-dependent kinases, $\mathrm{pRb}$, and $\mathrm{E} 2 \mathrm{~F} \cdot \mathrm{DP}$, in B-amyloid-induced neuronal death. J Biol Chem 274:19011-19016.

Hammang JP, Behringer RR, Baetge EE, Palmiter RD, Brinster RL, Messing A (1993) Oncogene expression in retinal horizontal cells of transgenic mice results in a cascade of neurodegeneration. Neuron 10:1197-1209.

Herrup K, Busser JC (1995) The induction of multiple cell cycle events precedes target-related neuronal death. Development 121:2385-2395.

Ino H, Chiba T (1996) Intracellular localization of cyclin-dependent kinase 5 (CDK5) in mouse neuron: CDK5 is located in both nucleus and cytoplasm. Brain Res 732:179-185.

Ino H, Chiba T (2000) Expression of proliferating cell nuclear antigen (PCNA) in the adult and developing mouse nervous system. Mol Brain Res 78:163-174.

Ino H, Ishizuka T, Chiba T, Tatibana M (1994) Expression of CDK5 (PSSALRE kinase), a neuronal cdc2-related protein kinase, in the mature and developing mouse central and peripheral nervous systems. Brain Res 661:196-206.

Kasof GM, Mandelzys A, Maika SD, Hammer RE, Curran T, Morgan JI (1995) Kainic acid-induced neuronal death is associated with DNA damage and a unique immediate-early gene response in c-fos-lacZ transgenic rats. J Neurosci 15:4238-4249.

Kaya SS, Mahmood A, Yayuz E, Chopp M (1999) Expression of cell cycle proteins (cyclin D1 and cdk4) after controlled cortical impact in rat brain. J Neurotrauma 16:1187-1196.

Kranenburg O, van der Eb A, Zantema A (1996) Cyclin D1 is an essential mediator of apoptotic neuronal cell death. EMBO J 15:46-54.

Levkau B, Koyama H, Raines EW, Clurman BE, Herren B, Orth K, Roberts JM, Ross R (1998) Cleavage of p21 Cip1/Waf1 and p27 ${ }^{\text {Kip1 }}$ mediates apoptosis in endothelial cells through activation of $\mathrm{Cdk} 2$ : role of a caspase cascade. Mol Cell 1:553-563.

McShea A, Harris PLR, Webster KR, Wahl AF, Smith MA (1997) Abnormal expression of the cell cycle regulators p16 and CDK4 in Alzheimer's disease. Am J Pathol 150:1933-1939.

Mullen RJ, Buck CR, Smith AM (1992) NeuN, a neuronal specific nuclear protein in vertebrates. Development 116:201-211.

Neuman E, Ladha MH, Lin N, Upton TM, Miller SJ, DiRenzo J, Pestell RG, Hinds PW, Dowdy SF, Brown M, Ewen ME (1997) Cyclin D1 stimulation of estrogen receptor transcriptional activity independent of cdk4. Mol Cell Biol 17:5338-5347.

Osuga H, Osuga S, Wang F, Fetni R, Hogan MJ, Slack RS, Hakim AM, Ikeda J, Park DS (2000) Cyclin-dependent kinases as a therapeutic target for stroke. Proc Natl Acad Sci USA 97:10254-10259.

Park DS, Farinelli SE, Greene LA (1996) Inhibitors of cyclin-dependent kinases promote survival of post-mitotic neuronally differentiated PC12 cells and sympathetic neurons. J Biol Chem 271:8161-8169.

Park DS, Levine B, Ferrari G, Greene LA (1997) Cyclin dependent kinase inhibitors and dominant negative cyclin dependent kinase 4 and 6 promote survival of NGF-deprived sympathetic neurons. J Neurosci 17:8975-8983.

Park DS, Morris EJ, Bremner R, Keramaris E, Padmanabhan J, Rosenbaum M, Shelanski ML, Geller HM, Greene LA (2000) Involvement of retinoblastoma family members and E2F/DP complexes in the death of neurons evoked by DNA damage. J Neurosci 20:3104-3114.

Paxinos G, Watson C (1998) The rat brain in stereotaxic coordinates, 4th Edition. San Diego: Academic.

Pettmann B, Henderson CE (1998) Neuronal cell death. Neuron 20:633-647.

Sakurai M, Hayashi T, Abe K, Itoyama Y, Tabayashi K (2000) Cyclin D1 and $\mathrm{Cdk} 4$ protein induction in motor neurons after transient spinal cord ischemia in rabbits. Stroke 31:200-207.

Schlingensiepen K-H, Wollnik F, Kunst M, Schlingensiepen R, Herdegen T, Brysch W (1994) The role of Jun transcription factor expression and phosphorylation in neuronal differentiation, neuronal cell death, and plastic adaptations in vivo. Cell Mol Neurobiol 14:487-505.

Schwob JE, Fuller T, Price JL, Olney JW (1980) Widespread patterns of neuronal damage following systemic or intracerebral injections of kainic acid: a histological study. Neuroscience 5:991-1014.

Shi L, Nishioka WK, Th'ng J, Bradburry EM, Litchfield DW, Greenburg AH (1994) Premature p34 ${ }^{\mathrm{cdc} 2}$ activation required for apoptosis. Science 263:1143-1145. 
Smeyne RJ, Vendrell M, Hayward M, Baker SJ, Miao GG, Schilling K, Robertson LM, Curran T, Morgan JI (1993) Continuous c-fos expression precedes programmed cell death in vivo. Nature 363:166-169.

Streit WJ (1990) An improved staining method for rat microglial cells using the lectin from Griffonia simplicifolia $\left(\mathrm{GSA} I-\mathrm{B}_{4}\right)$. J Histochem Cytochem 38:1683-1686.

Timsit S, Rivera S, Ouaghi P, Guischard F, Tremblay E, Ben-Ari Y, Khrestchatisky M (1999) Increased cyclin D1 in vulnerable neurons in the hippocampus after ischemia and epilepsy: a modulator of in vivo programmed cell death? Eur J Neurosci 11:263-278.

van Lookeren Campagne M, Gill R (1998) Cell cycle-related gene expression in the adult rat brain: selective induction of cyclin $\mathrm{G}_{1}$ and $\mathrm{p} 21^{\mathrm{WAF} 1 / \mathrm{CIP} 1}$ in neurons following focal cerebral ischemia. Neuroscience 84:1097-1112.

Whitesell L, Geselowitz D, Chavany C, Fahmy B, Walbridge S, Alger JR, Neckers LM (1993) Stability, clearance, and disposition of intraven- tricularly administered oligodeoxynucleotides: implications for therapeutic application within the central nervous system. Proc Natl Acad Sci USA 90:4665-4669.

Wisdom R, Johnson RS, Moore C (1999) c-Jun regulates cell cycle progression and apoptosis by distinct mechanisms. EMBO J 18:188-197.

Yang DD, Kuan C-Y, Whitmarsh AJ, Rincón M, Zheng TS, Davis RJ, Rakic P, Flavell RA (1997) Absence of excitotoxicity-induced apoptosis in the hippocampus of mice lacking the Jnk3 gene. Nature 389:865-870.

Zhou B-B, Li H, Yuan J, Kirschner MW (1998) Caspase-dependent activation of cyclin-dependent kinases during Fas-induced apoptosis in Jurkat cells. Proc Natl Acad Sci USA 95:6785-6790.

Zwijsen RM, Wientjens E, Klompmaker R, van der Sman J, Bernards R, Michalides RJ (1997) CDK-independent activation of estrogen receptor by cyclin D1. Cell 88:405-415. 